\title{
Microbiome analysis of Pacific white shrimp gut and rearing water from Malaysia and Vietnam: Implications for aquaculture research and management
}

\author{
Muhammad Zarul Hanifah MdZoqratt ${ }^{1,2}$, Wilhelm Wei Han Eng ${ }^{1,2}$, Binh Thanh Thai ${ }^{3}$, Christopher M Austin ${ }^{1}$ \\ 2,4, Han Ming Gan Corresp. 1, 2,4 \\ ${ }^{1}$ School of Science, Monash University Malaysia, Petaling Jaya, Selangor, Malaysia \\ 2 Genomics Facility, Tropical Medicine and Biology Platform, Monash University Malaysia, Petaling Jaya, Selangor, Malaysia \\ 3 Fisheries and Technical, Economical College, Dinh Bang, Tu Son, Vietnam \\ ${ }^{4}$ Centre for Integrative Ecology, School of Life and Environmental Sciences, Deakin University, Geelong, Victoria, Australia \\ Corresponding Author: Han Ming Gan \\ Email address: han.gan@deakin.edu.au
}

Aquaculture production of the Pacific white shrimp is the largest in the world for crustacean species. Crucial to the sustainable global production of this important seafood species is a fundamental understanding of the shrimp gut microbiota and its relationship to the microbial ecology of shrimp pond. This is especially true, given the recently recognized role of beneficial microbes in promoting shrimp nutrient intake and in conferring resistance against pathogens. Unfortunately, aquaculture related microbiome studies are scarce in Southeast Asia countries despite the severe impact of early mortality syndrome outbreaks on shrimp production in the region. In this study, we employed the 16S rRNA amplicon (V3-V4 region) sequencing and amplicon sequence variants (ASV) method to investigate the microbial diversity of shrimp guts and pond water samples collected from aquaculture farms located in Malaysia and Vietnam. Substantial differences in the pond microbiota were observed between countries with the presence and absence of several taxa extending to the family level. Microbial diversity of the shrimp gut was found to be generally lower than that of the pond environments with a few ubiquitous genera representing a majority of the shrimp gut microbial diversity such as Vibrio and Photobacterium, indicating host-specific selection of microbial species. Given the high sequence conservation of the 16S rRNA gene, we assessed its veracity at distinguishing Vibrio species based on nucleotide alignment against type strain reference sequences and demonstrated the utility of ASV approach in uncovering a wider diversity of Vibrio species compared to the conventional OTU clustering approach. 
1 Microbiome analysis of Pacific white shrimp gut and rearing water from Malaysia and

2 Vietnam: Implications for aquaculture research and management

4 Muhammad Zarul Hanifah MdZoqratt ${ }^{1,2}$, Wilhelm Wei Han Eng ${ }^{1,2}$, Binh Thanh Thai ${ }^{3}$, 5 Christopher M. Austin 1,2,4, Han Ming Gan1,2,4 *

$6{ }^{1}$ Genomics Facility, Tropical and Medicine Biology Platform, Monash University Malaysia,

7 Petaling Jaya, Malaysia

$8{ }^{2}$ School of Science, Monash University Malaysia, Petaling Jaya, Malaysia

$9 \quad{ }^{3}$ Fisheries and Technical, Economical College, Dinh Bang, Tu Son, Vietnam

${ }^{4}$ Centre for Integrative Ecology, School of Life and Environmental Sciences, Deakin University,

11 Geelong, Australia

12

\title{
* Correspondence:
}

Han Ming Gan

han.gan@deakin.edu

\begin{abstract}
Aquaculture production of the Pacific white shrimp is the largest in the world for crustacean species. Crucial to the sustainable global production of this important seafood species is a fundamental understanding of the shrimp gut microbiota and its relationship to the microbial ecology of shrimp pond. This is especially true, given the recently recognized role of beneficial microbes in promoting shrimp nutrient intake and in conferring resistance against pathogens. Unfortunately, aquaculture related microbiome studies are scarce in Southeast Asia countries despite the severe impact of early mortality syndrome outbreaks on shrimp production in the region. In this study, we employed the 16S rRNA amplicon (V3-V4 region) sequencing and amplicon sequence variants (ASV) method to investigate the microbial diversity of shrimp guts and pond water samples collected from aquaculture farms located in Malaysia and Vietnam. Substantial differences in the pond microbiota were observed between countries with the
\end{abstract}


32 presence and absence of several taxa extending to the family level. Microbial diversity of the

33 shrimp gut was found to be generally lower than that of the pond environments with a few ubiquitous genera representing a majority of the shrimp gut microbial diversity such as Vibrio and Photobacterium, indicating host-specific selection of microbial species. Given the high sequence conservation of the $16 \mathrm{~S}$ rRNA gene, we assessed its veracity at distinguishing Vibrio species based on nucleotide alignment against type strain reference sequences and demonstrated the utility of ASV approach in uncovering a wider diversity of Vibrio species compared to the conventional OTU clustering approach.

\section{Introduction}

Litopenaeus vannamei (Boone, 1931), also known as the Pacific white shrimp or Whiteleg shrimp, is a major aquaculture commodity with a production of 3.69 million tonnes valued at 18 billion USD revenue (FAO, 2016). In recent years, significant outbreaks of acute hepatopancreatic necrosis disease (AHPND), also known as early mortality syndrome (EMS) have been reported in a number of white shrimp-producing countries. EMS was first reported in China in 2009 and subsequently spread to Southeast Asian countries including Vietnam, Malaysia, and Thailand (Foo et al. 2017; Kondo et al. 2014; Tran et al. 2013). The causative agent of EMS has been reported to be Vibrio parahaemolyticus strains harbouring a plasmid containing the pirA-and pirB-like genes encoding for toxins capable of severely damaging the shrimp gut (Han et al. 2015; Lee et al. 2015). To date, EMS has caused an estimated 1 billion USD of losses to the shrimp industry worldwide (De Schryver et al. 2014; Lee et al. 2015). Monitoring and control of pond water quality play a crucial role in managing and preventing disease outbreak in aquaculture. However, the current practice of water quality monitoring usually focuses on the measurement of chemical and physical parameters such as oxygen, $\mathrm{pH}$, temperature, salinity, turbidity and nitrogen compounds. The importance of microbial communities in influencing or responding to variation aquaculture pond water quality has only been recognized in recent years (Bentzon-Tilia et al. 2016). This is especially relevant to managing the water quality of aquaculture ponds and their cultured biomass because microbes carry out important biological services in aquaculture environment including nutrient cycling, probiotic/pathogenic activity and nutrient acquisition in addition to potentially acting as a rapid 
63 biological indicator of critical chemical changes in the rearing water (Cardona et al. 2016;

64 Cornejo-Granados et al. 2017; Costa et al. 2006; Emerenciano et al. 2013; Grotkjær et al. 2016;

65

66

67 Jinbo et al. 2017; Liu et al. 2015; Wright et al. 2012; Zeng et al. 2017; Zhu et al. 2016). Microbes can also be used to improve the water quality of ponds. For example, adding denitrifying bacteria to biofilters has been shown to reduce the concentration of ammonia and its immediate derivatives, which are detrimental to shrimp health (Saffran et al. 2001).

Recognizing the importance of microbial biomass and diversity on the health and production of cultured invertebrates, several microbiome studies have analysed the gut microbiome of wild-caught shrimps (Cornejo-Granados et al. 2017; Phayungsak et al. 2018; Rungrassamee et al. 2016) as well as cultured shrimps under different abiotic and biotic factors. However, most studies have been restricted to a specific country especially China, focusing only on one or very few localized ponds (Cornejo-Granados et al. 2017; Huang et al. 2016; Rungrassamee et al. 2016; Tang et al. 2014; Xiong et al. 2015; Zhang et al. 2014; Zhu et al. 2016). A study in Thailand used denaturing gradient gel electrophoresis profiling and barcoded pyrosequencing to demonstrate a greater survivability of Litopenaeus vannamei and improved the resilience of its gut microbiome upon Vibrio harveyi exposure relative to that of Penaeus monodon (Rungrassamee et al. 2016). Another more recent study in Vietnam utilised a standard Illumina 16S rRNA gene amplicon sequencing method to investigate the effect of EMS outbreak on the microbial interaction networks in shrimp guts (Chen et al. 2017b). Thus, despite the emergence of Southeast Asia (SEA) as an aquaculture hub, studies on any significant geographic scale are relatively scarce in this region. Further, to our knowledge, all recent shrimp aquaculture microbiome studies still employ the operational taxonomic units (OTU) clustering approach in marker-gene data analysis despite recent call for the replacement of this approach with exact sequence variants which can resolve single-base differences among biological sequences in the sample thus providing a more comprehensive and accurate view of microbial community (Callahan et al. 2017; Eren et al. 2014; Utter et al. 2016).

To initiate a more broad-based investigation of shrimp microbiome directly relevant the aquaculture industry in SEA, we performed Illumina $16 \mathrm{~S}$ rRNA gene amplicon sequencing of $L$. vannamei guts and rearing water from aquaculture farms located in two SEA countries with contrasting climates at the time of sampling i.e. Malaysia (warm and humid, $30^{\circ} \mathrm{C}$ ) and Vietnam (cool and dry, $20^{\circ} \mathrm{C}$ ). For the first time in a shrimp aquaculture microbiome study, we applied the 
94

95

96

97 98

99

100

101

102

103

104

105

106

107

108

109

110

111

112

113

114

115

116

117

118

119

120 GTCTCGTGGGCTCGGAGATGTGTATAAGAGACAGGACTACHVGGGTATCTAATCC-

121 3' containing partial Illumina Nextera adapter. PCR reaction ( $\sim 10 \mathrm{ng}$ input DNA/ reaction) and 122 barcode incorporation were performed as previously described (Watts et al. 2017). Constructed 123 libraries were quantified, normalized, pooled, denatured and subsequently sequenced on the

recently advocated ASV-method (Edgar 2016) to (1) compare shrimp intestinal microbial diversity and their pond environments (2) compare these microbial communities between Malaysia and Vietnam and, (3) assess the performance of the 16S rRNA V3-V4 hypervariable region and clustering approaches in capturing the genetic diversity of the Vibrio.

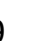

\section{Methods}

\section{Sample Collection}

Sampling in Vietnam was performed at two separate shrimp farms in the Quang Ninh province (approximately $20 \mathrm{~km}$ apart), while sampling in Malaysia was performed at a large shrimp farm with multiple pond systems located in Perak state (Table 1). $2 \mathrm{~mL}$ of pond water was sampled from 2-4 distant location (corners) of each pond, pelleted via centrifugation at 7,000 rpm for 10 minutes and resuspended in RNA/DNA shield (ZymoResearch, Irvine, CA, USA). Shrimp intestinal samples were collected by dissecting out the shrimp guts followed by homogenization in RNA/DNA shield (ZymoResearch, Irvine, CA, USA). Sampling was performed with the permission and under the supervision of the aquaculture manager from the respective farms. No field permit was required for this study because samples were collected from private fields. As per the request of the aquaculture manager, exact sampling location of some farms was not disclosed in this study to protect the identity of the farm.

\section{DNA Extraction, Amplification, Purification and Sequencing}

Genomic DNA was extracted from the RNA/DNA shield lysate using DNA Clean \& Concentrator ${ }^{\mathrm{TM}}-5$ (ZymoResearch, Irvine, CA, USA) according to the manufacturer's instructions. The V3-V4 region of the 16S rRNA gene was amplified using forward primer 5'TCGTCGGCAGCGTCAGATGTGTATAAGAGACAGCCTACGGGNGGCWGCAG-3' and reverse primer $5^{\prime}-$ 
124 Illumina MiSeq (Illumina, San Diego, CA, USA) located at Monash University Malaysia

125 Genomics Facility using a $2 \times 250$ bp run configuration.

126

127 Sequence Data Analysis and Observation Table Construction

128 Primer sequences corresponding to the $16 \mathrm{~S}$ rRNA gene were removed from the raw paired-end 129 reads using Cutadapt (Martin 2011). Trimmed forward and reverse reads were overlapped with 130 fastq mergepairs followed by quality and length filtering with fastq filter (maximum expected error $=0.5$; min length $=250 \mathrm{bp})$ as implemented in USearch10 (Edgar 2010). Sequence dereplication and denoising was done using uNoise 3 to generate amplicon sequence variants (ASVs) (Edgar 2016). Aside from "-minuniquesize 2" parameter during sequence dereplication, the processes of the pipeline were done using default parameters. Taxonomy assignment and observation table construction rarefied at 8,000 reads were performed in RDP classifier 2.12 and QIIME 1.9.1 (Caporaso et al. 2010; Cole et al. 2009). ASVs that failed RDP taxonomic assignment were re-classified using SINA 1.3.1 against SILVA SSU Ref database (release 132) with default parameters (Supplementary Data 3 and 4) (Pruesse et al. 2012; Pruesse et al. 2007). ASVs with lower than $0.01 \%$ fraction of the total normalized observation and/or identified as chloroplast were not included in subsequent analyses. Normalization of sequencing depth per sample (8,000 reads/sample), rarefaction curves construction (10 replicates/depth) as well as alpha diversity estimation (Simpson's evenness and Shannon diversity indices), were performed using the "core_diversity.py" python script in QIIME 1.9.1. Core genera of the shrimp gut microbiome were investigated and defined as genera with at least $0.1 \%$ relative abundance per sample in more than $50 \%$ shrimp samples. The prevalence and relative abundance of the shrimp gut core genera were visualised using the R ggplot2 package (Wickham \& Wickham 2007).

\section{Principal Coordinates Analysis and Relative Differential Abundance Analysis}

The proportion of sequences assigned to the lowest possible taxonomic level was calculated based on the rarefied observation table and the implemented taxonomic assignment method mentioned above. Principal coordinates analysis (PCoA) was also constructed based on weighted Unifrac and unweighted Unifrac using ordination method in $\mathrm{R}$ phyloseq package (Lozupone \& 153 Knight 2005; McMurdie \& Holmes 2013). The resulting PCoA plots were then visualized using 
155 calculated using the compare_categories.py python script in QIIME which implements ANOSIM

156 analysis using the default 999 permutations. Relative differential abundance test was also

157 conducted at phylum and family levels using Tukey-Kramer post-hoc in conjunction with

158 analysis of variance (ANOVA) statistical test in STAMP (Parks et al. 2014). Multiple hypothesis

159 testing was done for the four generic groups namely Malaysian Farm, Malaysian Shrimp,

160 Vietnamese Farm and Vietnamese Shrimp. A Significant differential abundance of phylum

161 distribution was defined as Benjamini-Hochberg-corrected probability $p$-value of $\leq 0.01$ and was

162 observed only for the top 10 most abundant phyla. A significant differential abundance of family

163 distribution was defined as Benjamini-Hochberg corrected $p$-value of $\leq 0.01$ and eta squared $\geq$

$164 \quad 0.3$.

165

166 Comparison of Vibrio Diversity Using Different Clustering Methods

167 Vibrio diversity was compared using different methods of 16S marker-gene data analysis,

168 namely conventional operational taxonomic unit (OTU) clustering and amplicon sequence

169 variants (ASV), using UParse and uNoise3 respectively (Edgar 2013; Edgar 2016). Except for -

170 minuniquesize of 2 during sequence dereplication, both pipelines were conducted using default

171 parameters. ASVs and OTUs assigned to the genus Vibrio with at least cumulative read

172 abundance of more than 200 were retained for blastN similarity search (E-value $<1$ e-100)

173 against 16S rRNA sequences of Vibrio type strain curated in EzBioCloud (as of 18 ${ }^{\text {th }}$ May 2018)

174 (Yoon et al. 2017)

175

176 Results

177 Shrimp intestinal and pond microbial communities are distinct

178 A total of 2,731,818 successfully merged reads were generated in this study with 2,144,192 reads

179 (median of 32,648 reads/sample; $\min =9,948 ; \max =66,590$ ) confidently mapped to the ASVs.

$18092.12 \%$ and $7.77 \%$ of the mapped reads correspond to RDP-classified and SINA-classified ASVs

181 respectively, while the remaining mapped reads belong to ASVs without confident taxonomic

182 assignment at the kingdom rank. A majority of reads recovered from shrimp intestine and ponds

183 were assigned to members from the phyla Proteobacteria, Actinobacteria, Bacteroidetes and

184 Fusobacteria (Figure 1). Significant differences in the relative abundance of bacteria phyla were

185 observed among samples isolated from ponds and shrimp guts. Reads mapping to Actinobacteria 
186

187

188

189

190

191

192

193

194

195

196

197

198

199

200

201

202

203

204

205

206

207

208

209

210

211

212

213

214

215

216

and Bacteroidetes are more abundant in ponds $(\mathrm{p}<0.01$ and $\mathrm{p}<0.001$, respectively), while shrimp guts have a significantly higher relative abundance of Proteobacteria $(\mathrm{p}<0.001)$. At a finer level, Malaysian shrimp intestinal microbiome contains more reads mapping to the phylum Fusobacteria $(\mathrm{p}<0.01)$. Notable, this phylum is also near absent in 3 out of 4 Malaysian shrimps noted to be unhealthy based on morphological observation by the aquaculture manager (Figure $1)$.

Rarefaction curves based on alpha diversity metrics, number of observed ASVs and PD (Phylogenetic diversity) whole tree, indicated that 8,000 sequences per sample are sufficient for capturing the alpha diversity of microbial communities in both shrimp guts and ponds. Inverse Simpson's and Shannon's indices showed higher species richness and evenness in the pond microbiome compared to that of shrimp gut (Figure 2A and 2B). Beta diversity analyses based on both weighted and unweighted UniFrac indicated that shrimp gut and pond microbial communities from the same sampling site are significantly different (Vietnam : $\mathrm{R}>0.67$, p-value $<0.001$; Malaysia: $\mathrm{R}>0.89$, p-value $<0.001$ ) (Supplementary Table 2). An even stronger separation was also observed among pond samples from different sampling sites/ countries (Figure 3, Supplementary Table 2). Furthermore, samples from one of the Malaysian ponds (MF_Pond11) noted by the shrimp farmer to be infested by mussels (Supplementary Table 1) was distinct from other Malaysian pond sample samples (Figure 3). It is worth noting that rearing water samples collected from different parts of the same pond have minimal spatial variation in microbial composition as evidenced by the general tight clustering of pond replicates, suggesting homogenous microbial community in the rearing water and indicating that the sampling protocols are efficient for capturing pond diversity. Although the separation between Malaysian and Vietnamese shrimp gut samples was less obvious in both PCoA plots with occasional overlap, their microbial community structure appears to differ moderately $(\mathrm{R}<0.67)$ with good statistical support ( $\mathrm{p}$-value $<0.001$ ) based on ANOSIM analysis (Supplementary Table 2).

\section{Comparison of relative abundance at the microbial family level revealed fine-level} microbiota dynamics

Given that more than $70 \%$ and $85 \%$ of the reads derived from pond and shrimp intestinal samples, respectively, could be assigned to the family level (Supplementary Data S3 and S4), we 
217 defined the core and unique microbiomes among sample groups at this taxonomic level and used

218 STAMP to identify statistically significant differences in the relative abundance of microbial 219 families. A total of 6 microbial families (Alcaligenaceae, Flavobacteriaceae, Microbacteriaceae, 220 Acidimicrobiaceae and Rhodobacteraceae) are shared across shrimp and rearing water samples

221 (Figure 4A and Supplementary Data S5) with 7 and 10 microbial families uniquely present in

222 Malaysian and Vietnamese rearing water, respectively, corroborating with their higher alpha

223 diversity (Figure 2C and 2D). In addition, 4 microbial families are uniquely shared by the

224 Malaysian and Vietnamese rearing water samples, suggesting their common affiliation with

225 shrimp rearing water. Certain microbial families are significantly more abundant in samples of

226 the same isolation source, regardless of the country of origin. For example, Microbacteriaceae

227 and Flavobacteriaceae are more abundant in pond water, while Vibrionaceae is highly enriched

228 in the shrimp gut (Figure 4B). On the other hand, Rhodobacteraceae is more abundant in

229 Vietnamese samples (shrimp gut and rearing water) while Cyanobacteria-Family II is more

230 abundant in Malaysian samples, suggesting possible affiliation to regional climate and/or pond

231 environment. In addition, four microbial families (Acidomicrobiaceae, Actinomarinaceae,

232 Comamonadaceae, and Fusobacteriaceae) showed significantly higher abundance only in a

233 specific country and isolation source (Figure 4B).

234 High prevalence and relative abundance of ASVs belonging to the genera Vibrio in shrimp

235 gut

236

237 16S rRNA reads corresponding to 11 bacterial genera belonging to 5 phyla (Actinobacteria,

238 Chloroflexi, Cyanobacteria, Planctomycetes and Proteobacteria) were detected in more than 50\%

239 of the shrimp gut samples (Figure 5) with Vibrio being the only genera present in all shrimp gut

240 samples with relatively similar relative abundance across samples(mean/median relative

241 abundance per sample $=33.7 \% / 27.7 \%$ ). On the contrary, Photobacterium, a genus related to

242 Vibrio at the family level (Vibrionaceae) was detected in all but two Vietnamese shrimps

$243($ mean $/$ median relative abundance per sample $=11.6 \% / 6.5 \%)$. The prevalence of the core genera

244 was independently correlated with relative abundance e.g. the higher the prevalence of a core

245 genus, the more likely it is to have a higher relative abundance. Rhodopirellua and Gimesia all

246 belonging to the phylum Planctomycetes are more prevalent and abundant in Vietnamese

247 shrimps than in the Malaysian shrimps, consistent with statistical analysis showing a 
248 significantly higher abundance of Planctomycetes in Vietnamese shrimps compared to Malaysian

249 shrimps (Figure 1).

250

251

252

Substantial underestimation of Vibrio diversity using the OTU clustering approach

The high cumulative abundance of reads assigned to the genus Vibrio in shrimp guts indicates

253

that some members of this genus are endogenous to the shrimp gut microbiota. UPARSE using

254 the default $97 \%$ sequence similarity cut-off setting identified two abundant OTUs assigned to

255 Vibrio. This contrasts greatly with the ASV approach which identified substantially more biological sequences classified as Vibrio (Figure 6, Supplementary Data 3 and 4). A majority of the constructed ASVs do not have an exact sequence match to the constructed OTUs and more importantly, some could be assigned to a single Vibrio species (ASV22, Vibrio jasicida TCFB $0772^{\mathrm{T}}$; ASV19, Vibrio neocaledonicus $\mathrm{NC470}^{\mathrm{T}}$ ). OTU2 and OTU10 are identical in both sequence length and identity to ASV2 and ASV14, respectively. Similarity search of

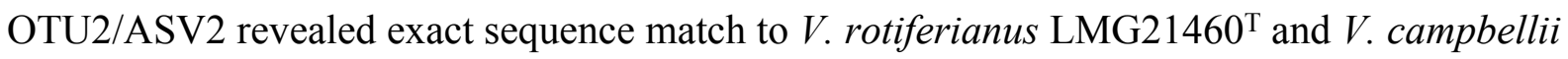
CAIM519 ${ }^{\mathrm{T}}$, indicating a limitation to the use of V3-V4 hypervariable region in delimiting some Vibrio species (Figure 6). The high ratios of ASVs-to-OTUs observed for OTU2 and OTU10

264 265

266

267 strongly suggests that imposing a fixed dissimilarity threshold using conventional OTU clustering underestimates the true microbial diversity for Vibrio species in this study and that resolving amplicon sequence variants (ASV) from amplicons data which is sensitive down to single-nucleotide differences, through read de-replication and error correction, substantially increased the number of observed Vibrio species from the identical dataset. Unlike the ASVs associated with OTU2, nearly all ASVs associated with OTU10 have at least 2 mismatches to known Vibrio species, indicating the presence of unculturable or yet-to-be-cultured Vibrio strains in the shrimp gut. Of even more interest, the ASV approach revealed the presence of $V$. parahaemolyticus (ASV235 in Figure 6) that was missed by the OTU clustering approach presumably due to its overall low relative abundance across samples (Supplementary Table 5).

\section{Discussion}

Despite the immense scale of shrimp aquaculture in South East Asia and the major impacts of aquaculture disease outbreaks in tropical regions, we are only starting to understand the microbial composition of the shrimp guts and their relationship to rearing water in the region 
279 (Leung \& Bates 2013). Most shrimp-related microbiome studies have been limited to a few

280 farms in a particular country; most of which have been conducted in countries outside of SEA

281 such as China and Mexico. Litopenaeus vannamei microbiome studies have so far investigated

282 the microbial composition of wild-type shrimps serving as an important baseline for future

283 comparative studies(Cornejo-Granados et al. 2017) as well as the impacts of disease exposure

284 (Chen et al. 2017b; Cornejo-Granados et al. 2017; Jinbo et al. 2017; Rungrassamee et al. 2016;

285 Xiong et al. 2015; Zhu et al. 2016), developmental stages (Huang et al. 2016), nutrition (Zhang

286 et al. 2014) and temperature (Tang et al. 2014) on shrimp intestinal microbiome. We have

287 contributed new findings to the growing literature by providing first data on gut and pond water

288 microbiome of Malaysian cultured shrimps. Furthermore, we compared bacterial communities of

289 shrimp guts and pond water from multiple aquaculture farms in two distinct climatic regions

290 (Malaysia and north Vietnam) while standardizing DNA extraction and sequencing protocols.

291 This provides a new perspective on our current understanding on the range of normal microbial

292 composition of a healthy shrimp gut microbiome community. Our principal findings provide

293 evidence supporting microbiota plasticity in shrimp ponds, but in contradistinction, find a much

294 more limited diversity of the adult shrimp intestinal microbiota.

Comparison of the pond water microbiome between the Malaysian and Vietnamese

296 samples reveals significant dissimilarities at the phylum level as shown in Figure 1. Although

297 Planctomycetes was identified as a significant phylum in this study, it was not commonly found

298 in other metagenomic studies of similar environments (Li et al. 2016; Xiong et al. 2015; Zeng et

299 al. 2017) (Supplementary Table 4). The only sample site with near-zero relative abundance of

300 Plantomycetes was a mussel-infested Malaysian aquaculture pond (Pond11 in Figure 1). Mussels

301 are known ecosystem engineers that can substantially modify their habitats through biological

302 processes such as sediment filtration and biodeposition of fecal matters (Bril et al. 2014). The

303 presence of mussels has been reported to cause to a 4-fold decrease in the relative abundance of

304 Plantomycetes in a freshwater system in Mississippi, USA (Black et al. 2017).

305 Although Flavobacteriaceae and Microbacteriaceae are prevalent in the rearing water and

306 shrimp gut samples, they exhibited significantly higher relative abundance in the rearing water

307 samples. Albeit initially associated with a fairly general ecological function e.g. simple

308 mineralization-based commensalism (Kirchman 2002), emerging evidence suggests that some

309 members within the marine Flavobactericeae clade are algal-associated species that exhibit 
310 growth promoting and inhibiting effects to its host and other algal species, respectively (Bowman

311 2006). Genera such as Cellulophaga, Psychroserpens and Formoasa have been previously

312 reported to produce toxic secondary metabolites against dinoflagellates, commonly associated

313 with algal bloom (Adachi et al. 2002; Egan et al. 2000). Thus, the significant abundance of

314 Flavobacteriacea in both rearing water could be linked to the natural occurrence of algal and

315 diatom species in the rearing water some of which were co-amplified by the V3-V4 primers in

316 this study (Supplementary Data 3 and Supplementary Table 3). On the contrary, most described

317 members from the family Microbacteriaceae were not associated with marine environmental and

318 were typically isolated from the terrestrial environment (Evtushenko \& Takeuchi 2017). High

319 abundance of Microbacteriaceae (unclassified at the genus level) in shrimp rearing water

320 particularly during the post-larvae stage has been previously observed in a commercial marine

321 shrimp hatchery in Hainan, China (Zeng et al. 2017) which was suggested to be a temporal-

322 specific bacterial family caused by changes in the shrimp diet during different growth stages. On

323 the contrary, four microbial families namely, Cryomorphaceae, Rhodospirillaceae,

324 Bacteriovoracaceae and Saprospiraceae, are exclusively found across both Malaysian and

325 Vietnamese shrimp rearing water samples, indicating their specific adaptation to shrimp rearing

326 water or more generally the marine aquatic environment. For example, members of the family

327 Rhodospirillaceae are purple non-sulfur and mostly nitrogen-fixing photosynthetic bacteria.

328 Their absence in the shrimp gut is consistent with their strict requirement for light to grow and

329 proliferate which is not sufficiently present in the shrimp gut environment.

330 Despite the conspicuous difference in the shrimp pond microbiota between two countries,

331 the shrimp intestinal microbiota are more similar to each other which is presumably due to host

332 selection for microbial strains that adapt to or exploit the shrimp gut environment as corroborated

333 by their lower alpha diversity indices compared to that of rearing water samples (Xiong et al.

334 2017). However, the presence of several microbial families in both shrimp and rearing water

335 samples indicates that the shrimp gut microbiota maybe significantly affected by the microbial

336 communities present in their aquatic environment e.g. rearing water and pond sediments (Chen et

337 al. 2017a; Cornejo-Granados et al. 2017) as opposed to being maternally influenced as observed

338 in some mammals and other animals with forms of parental care (Jakobsson et al. 2014; Kohl \&

339 Dearing 2012; Zhang et al. 2014). Moulting e.g. shedding of shrimp ectodermal gut tissue also

340 provides a new opportunity for shrimp stomach and guts to be colonised by the bacterial 
341 community of the pond (Moss et al. 2000). Furthermore, crustaceans, including L. vannamei,

342 also consume their exuvia, which provides another opportunity for microbial recolonization of

343 the shrimp guts and also the transmission of intestinal microbiome across shrimps throughout

344 their developmental stages (Martínez-Córdova \& Peña-Messina 2005).

345 Although the micro-clustering of shrimp gut samples based on country and/or farm of

346 origin may be associated with the difference in their respective growth environment, variation in

347 developmental stage may also contribute to the observed clustering as the shrimps in this study

348 were collected at different adult growth stages (Table 1). The abundance of members from the

349 family Fusobacteriaceae is highly dependent on the shrimp developmental stage with a near-zero

350 abundance in young shrimp larvae gut and subsequently making up a substantial portion of the

351 microbiome in the adult stage (75-day post-hatching) (Chen et al. 2017b; Zeng et al. 2017).

352 Intriguingly, although most of the Vietnamaese shrimps were 97-day post-hatching during

353 sampling, the low abundance and prevalence of Fusobactericeae in the their guts indicates

354 microbiome resemblance to that of younger shrimps (Zeng et al. 2017). In contrast,

355 Fusobacteriaceae is prevalent in Malaysian shrimps that are relatively young e.g. 65-day post-

356 hatching. However, it is worth noting that Sitiawan, the closest city to where the Malaysian farm

357 is located, is warm $\left(30^{\circ} \mathrm{C}\right)$ throughout the year. Such a climate may support faster shrimp

358 growth thus enabling them to reach adulthood earlier (Kumlu et al. 2010; Wyban et al. 1995).

359 Members from the family Fusobacteriaceae are microaerotolerant to obligate anaerobic, Gram-

360 negative rods bacteria that derive energy through the fermentation of a variety of carbohydrates,

361 amino acids and peptides. Such a metabolic profile is consistent with the higher prevalence and

362 abundance of Fusobacteriaceae in mature shrimp intestinal systems that typically exhibit a better

363 digestive ability (Parte et al. 2011; Schock et al. 2013). Unfortunately, despite exhibiting a wide

364 ecological diversity as evidenced by their diverse isolation source, the symbiotic relationship of

365 Fusobacteriaceae towards its host is has yet been properly demonstrated (Nelson et al. 2013).

366 Future work, consisting of metatranscriptome and metagenome sequencing of the shrimp gut

367 microbiota will be necessary to shed light on the role of Fusobactericeae in the shrimp gut.

368 Vibrio and Photobacterium belonging to the Vibrionaceae family are both abundant and

369 prevalent in nearly all of the shrimp samples, an observation that is consistent with previous

370 reports (Cornejo-Granados et al. 2017; Rungrassamee et al. 2016; Xiong et al. 2017). In contrast,

371 Zeng et al. (2017) did not identify any Vibrio-specific OTUs in their sampling. Such anomalies 
372 are unlikely to be biological but rather due to technical and analytical differences, such as the

373 choice of the 16S rRNA gene region sequenced (V4- vs. V3-V4-hypervariable region) and

374 bioinformatic analysis settings. High abundance and prevalence of Vibrio and Photobacterium

375 genera in shrimp guts suggest that they are more likely to be endogenous rather than pathogenic

376 strains (Kriem et al. 2015). However, this also reflects the persistence and adaptation of members

377 from these genera to the shrimp gut environments and may potentially explain the susceptibility

378 of shrimps to non-native pathogenic Vibrio and Photobacterium strains (Kondo et al. 2014;

379 Wang \& Chen 2006).

380 The use of ASVs reveals a wider diversity of Vibrio species, suggesting that previous

381 shrimp microbiome analyses that employed the common 97\% similarity cut-off for clustering

382 will risk masking the true Vibrio diversity in the shrimp gut (Callahan et al. 2017; Chen et al.

383 2017b). Fortuitously, despite the observed low resolution of the V3-V4 hypervariable region for

384 Vibrio species, this region appears to be distinct in $V$. parahaemolyticus, which exhibits at least 2

385 diagnostic nucleotides that are absent from all known type strains of Vibrio species (Figure 6).

386 The lack of an OTU with exact match to ASV235 suggests that analysis using the OTU

387 clustering approach will fail to report the presence of $V$. parahaemolyticus and/or undescribed

388 Vibrio strains sharing the same 16S rRNA gene sequence with $V$. parahaemolyticus if they are

389 present at low abundance in the dataset. This can have critical implications for aquaculture

390 microbial management especially in the early detection of $V$. parahaemolyticus infection. Since

391 shrimp gut can harbour both pathogenic and native Vibrio species, complementing 16S rRNA-

392 based amplicon sequencing with an alternative genetic marker such as $\mathrm{pyrH}$ may enable a more

393 accurate quantification of Vibrio diversity and abundance in aquaculture environment (Tall et al.

394 2013; Thompson et al. 2005). Given the high diversity of shrimp gut-associated Vibrio as

395 revealed for the first time by the ASV approach, shallow shotgun metagenome sequencing will

396 also be instructive to obtain species/strain-level taxonomic resolution of the abundant

397 endogenous microbes in shrimp guts particularly those belonging to the genera Vibrio and

398 Photobacterium.

399 Shrimp gut microbiomes vary due to biological differences (shrimp strains), differences 400 in environmental or farming practice (temperature, diet, probiotic, wild capture) or even biases

401 from different laboratory procedures such as the applied sequencing platform and the different 402 partial 16S sequence target (Cornejo-Granados et al. 2017; Tremblay et al. 2015). Considering 
403 the crucial functions undertaken by microbial communities and the potential use of the pond

404 microbiome for pond health surveillance, investment in measuring a wide variety of chemical

405 and physical parameters would allow us to better correlate the relationship between microbiomes

406 and rearing water quality and therefore improving our understanding of aquaculture

407 microbiomes.

408

\section{Conclusions}

410 Using a standardized Illumina 16S rRNA amplicons sequencing protocol, we report for the first 411 time, amplicon sequence variants (ASV)-based analysis of aquaculture rearing water and shrimp 412 gut microbiota from two South East Asia countries with different climates. Despite substantial 413 difference in the microbial composition of shrimp rearing water between farms in Malaysia and 414 Vietnam, adult shrimp guts are more similar and exhibit a genus level core microbiome with the 415 genus Vibrio being the most prevalent and abundant group. In addition, compared to OTU 416 clustering approach, the ASV method improved the identification of closely related and/or rare 417 Vibrio species, which is of relevance to the shrimp aquaculture industry. The high abundance of 418 Vibrio in shrimp gut also suggests that some Vibrio species are endogenous and non-virulent to 419 shrimps with functional and ecological roles that remain to be elucidated in the future.

420

421

\section{Data Access}

422 All FastQ raw data can be accessed through SRA accession SRP126985, or through NCBI BioProject under BioProject ID of PRJNA422950

424

\section{Acknowledgement}

We thank the Monash University Malaysia Genomics Facility for the provision of computational resources. We are also extremely grateful to the aquaculture managers for providing access to their farms and sharing information.

\section{References} Flavobacterium sp. 5 N-3 using multiple probes and whole-cell hybridization. Fisheries science 68:713-720. 
434

435

436

437

438

439

440

441

442

443

444

445

446

447

448

449

450

451

452

453

454

455

456

457

458

459

460

461

462

463

464

465

466

467

468

469

470

471

472

473

474

475

476

477

478

Bentzon-Tilia M, Sonnenschein EC, and Gram L. 2016. Monitoring and managing microbes in aquaculture-Towards a sustainable industry. Microbial biotechnology 9:576-584.

Black EM, Chimenti MS, and Just CL. 2017. Effect of freshwater mussels on the vertical distribution of anaerobic ammonia oxidizers and other nitrogen-transforming microorganisms in upper Mississippi river sediment. PeerJ 5:e3536. 10.7717/peerj.3536

Bowman JP. 2006. The Marine Clade of the Family Flavobacteriaceae: The Genera Aequorivita, Arenibacter, Cellulophaga, Croceibacter, Formosa, Gelidibacter, Gillisia, Maribacter, Mesonia, Muricauda, Polaribacter, Psychroflexus, Psychroserpens, Robiginitalea, Salegentibacter, Tenacibaculum, Ulvibacter, Vitellibacter and Zobellia. In: Dworkin M, Falkow S, Rosenberg E, Schleifer K-H, and Stackebrandt E, eds. The Prokaryotes: Volume 7: Proteobacteria: Delta, Epsilon Subclass. New York, NY: Springer New York, 677-694.

Bril JS, Durst JJ, Hurley BM, Just CL, and Newton TJ. 2014. Sensor data as a measure of native freshwater mussel impact on nitrate formation and food digestion in continuous-flow mesocosms. Freshwater Science 33:417-424. 10.1086/675448

Callahan BJ, McMurdie PJ, and Holmes SP. 2017. Exact sequence variants should replace operational taxonomic units in marker-gene data analysis. The ISME journal 11:2639.

Caporaso JG, Kuczynski J, Stombaugh J, Bittinger K, Bushman FD, Costello EK, Fierer N, Peña AG, Goodrich JK, and Gordon JI. 2010. QIIME allows analysis of high-throughput community sequencing data. Nature methods 7:335-336.

Cardona E, Gueguen Y, Magré K, Lorgeoux B, Piquemal D, Pierrat F, Noguier F, and Saulnier D. 2016. Bacterial community characterization of water and intestine of the shrimp Litopenaeus stylirostris in a biofloc system. BMC Microbiology 16:157. 10.1186/s12866016-0770-Z

Chen C-Y, Chen P-C, Weng FC-H, Shaw GT-W, and Wang D. 2017a. Habitat and indigenous gut microbes contribute to the plasticity of gut microbiome in oriental river prawn during rapid environmental change. PLOS ONE 12:e0181427. 10.1371/journal.pone.0181427

Chen W-Y, Ng TH, Wu J-H, Chen J-W, and Wang H-C. 2017b. Microbiome Dynamics in a Shrimp Grow-out Pond with Possible Outbreak of Acute Hepatopancreatic Necrosis Disease. Scientific reports 7:9395.

Cole JR, Wang Q, Cardenas E, Fish J, Chai B, Farris RJ, Kulam-Syed-Mohideen AS, McGarrell DM, Marsh T, Garrity GM, and Tiedje JM. 2009. The Ribosomal Database Project: improved alignments and new tools for rRNA analysis. Nucleic Acids Research 37:D141D145. 10.1093/nar/gkn879

Cornejo-Granados F, Lopez-Zavala AA, Gallardo-Becerra L, Mendoza-Vargas A, Sánchez F, Vichido R, Brieba LG, Viana MT, Sotelo-Mundo RR, and Ochoa-Leyva A. 2017. Microbiome of Pacific Whiteleg shrimp reveals differential bacterial community composition between Wild, Aquacultured and AHPND/EMS outbreak conditions. Scientific reports 7:11783.

Costa E, Pérez J, and Kreft J-U. 2006. Why is metabolic labour divided in nitrification? Trends in microbiology 14:213-219.

De Schryver P, Defoirdt T, and Sorgeloos P. 2014. Early mortality syndrome outbreaks: a microbial management issue in shrimp farming? PLoS pathogens 10:e1003919.

Edgar RC. 2010. Search and clustering orders of magnitude faster than BLAST. Bioinformatics 26:2460-2461. 
479

480

481

482

483

484

485

486

487

488

489

490

491

492

493

494

495

496

497

498

499

500

501

502

503

504

505

506

507

508

509

510

511

512

513

514

515

516

517

518

519

520

521

522

523

524

Edgar RC. 2013. UPARSE: highly accurate OTU sequences from microbial amplicon reads. Nature methods 10:996-998.

Edgar RC. 2016. UNOISE2: improved error-correction for Illumina 16S and ITS amplicon sequencing. bioRxiv:081257.

Egan S, Thomas T, Holmström C, and Kjelleberg S. 2000. Phylogenetic relationship and antifouling activity of bacterial epiphytes from the marine alga Ulva lactuca. Environmental Microbiology 2:343-347.

Emerenciano M, Gaxiola G, and Cuzon G. 2013. Biofloc technology (BFT): a review for aquaculture application and animal food industry. Biomass now-cultivation and utilization: InTech.

Eren AM, Morrison HG, Lescault PJ, Reveillaud J, Vineis JH, and Sogin ML. 2014. Minimum entropy decomposition: Unsupervised oligotyping for sensitive partitioning of highthroughput marker gene sequences. The Isme Journal 9:968. 10.1038/ismej.2014.195

\section{https://www.nature.com/articles/ismej2014195\#supplementary-information}

Evtushenko LI, and Takeuchi M. 2017. The Family Microbacteriaceae. Growth 18:2-35.

Foo SM, Eng WWH, Lee YP, Gui K, and Gan HM. 2017. New Sequence Types of Vibrio parahaemolyticus Isolated from a Malaysian Aquaculture Pond, as Revealed by WholeGenome Sequencing. Genome announcements 5. 10.1128/genomeA.00302-17

Grotkjær T, Bentzon-Tilia M, D'Alvise P, Dourala N, Nielsen KF, and Gram L. 2016. Isolation of TDA-producing Phaeobacter strains from sea bass larval rearing units and their probiotic effect against pathogenic Vibrio spp. in Artemia cultures. Systematic and applied microbiology 39:180-188.

Han JE, Tang KFJ, Tran LH, and Lightner DV. 2015. Photorhabdus insect-related (Pir) toxinlike genes in a plasmid of Vibrio parahaemolyticus, the causative agent of acute hepatopancreatic necrosis disease (AHPND) of shrimp. Diseases of aquatic organisms 113:33-40. 10.3354/dao02830

Huang Z, Li X, Wang L, and Shao Z. 2016. Changes in the intestinal bacterial community during the growth of white shrimp, Litopenaeus vannamei. Aquaculture Research 47:1737-1746.

Jakobsson HE, Abrahamsson TR, Jenmalm MC, Harris K, Quince C, Jernberg C, Björkstén B, Engstrand L, and Andersson AF. 2014. Decreased gut microbiota diversity, delayed Bacteroidetes colonisation and reduced Th1 responses in infants delivered by caesarean section. Gut 63:559-566.

Jinbo X, Jinyong Z, Wenfang D, Chunming D, Qiongfen Q, and Chenghua L. 2017. Integrating gut microbiota immaturity and disease-discriminatory taxa to diagnose the initiation and severity of shrimp disease. Environmental Microbiology 19:1490-1501. doi:10.1111/1462-2920.13701

Kirchman DL. 2002. The ecology of Cytophaga-Flavobacteria in aquatic environments. FEMS microbiology ecology 39:91-100.

Kohl KD, and Dearing M-D. 2012. Experience matters: prior exposure to plant toxins enhances diversity of gut microbes in herbivores. Ecology letters 15:1008-1015.

Kondo H, Tinwongger S, Proespraiwong P, Mavichak R, Unajak S, Nozaki R, and Hirono I. 2014. Draft genome sequences of six strains of Vibrio parahaemolyticus isolated from early mortality syndrome/acute hepatopancreatic necrosis disease shrimp in Thailand. Genome announcements 2:e0221-00214.

Kriem MR, Banni B, El Bouchtaoui H, Hamama A, El Marrakchi A, Chaouqy N, Robert-Pillot A, and Quilici ML. 2015. Prevalence of Vibrio spp. in raw shrimps (Parapenaeus 
longirostris) and performance of a chromogenic medium for the isolation of Vibrio strains. Lett Appl Microbiol 61:224-230.

Kumlu M, Türkmen S, and Kumlu M. 2010. Thermal tolerance of Litopenaeus vannamei (Crustacea: Penaeidae) acclimated to four temperatures. Journal of Thermal Biology 35:305-308. https://doi.org/10.1016/j.jtherbio.2010.06.009

Lee C-T, Chen I-T, Yang Y-T, Ko T-P, Huang Y-T, Huang J-Y, Huang M-F, Lin S-J, Chen C-Y, Lin S-S, Lightner DV, Wang H-C, Wang AH-J, Wang H-C, Hor L-I, and Lo C-F. 2015. The opportunistic marine pathogen Vibrio parahaemolyticus becomes virulent by acquiring a plasmid that expresses a deadly toxin. Proceedings of the National Academy of Sciences 112:10798-10803. 10.1073/pnas.1503129112

Leung TL, and Bates AE. 2013. More rapid and severe disease outbreaks for aquaculture at the tropics: implications for food security. Journal of Applied Ecology 50:215-222.

Li L, Yan B, Li S, Xu J, and An X. 2016. A comparison of bacterial community structure in seawater pond with shrimp, crab, and shellfish cultures and in non-cultured pond in Ganyu, Eastern China. Annals of microbiology 66:317-328.

Liu S, Ren H, Shen L, Lou L, Tian G, Zheng P, and Hu B. 2015. pH levels drive bacterial community structure in sediments of the Qiantang River as determined by 454 pyrosequencing. Frontiers in Microbiology 6.

Lozupone C, and Knight R. 2005. UniFrac: a new phylogenetic method for comparing microbial communities. Applied and Environmental Microbiology 71:8228-8235.

Martin M. 2011. Cutadapt removes adapter sequences from high-throughput sequencing reads. EMBnet journal 17:pp. 10-12.

Martínez-Córdova LR, and Peña-Messina E. 2005. Biotic communities and feeding habits of Litopenaeus vannamei (Boone 1931) and Litopenaeus stylirostris (Stimpson 1974) in monoculture and polyculture semi-intensive ponds. Aquaculture Research 36:1075-1084.

McMurdie PJ, and Holmes S. 2013. phyloseq: an R package for reproducible interactive analysis and graphics of microbiome census data. PLOS ONE 8:e61217.

Moss SM, LeaMaster BR, and Sweeney JN. 2000. Relative Abundance and Species Composition of Gram-Negative, Aerobic Bacteria Associated with the Gut of Juvenile White Shrimp Litopenaeus vannamei Reared in Oligotrophic Well Water and Eutrophic Pond Water. Journal of the World Aquaculture Society 31:255-263.

Nelson TM, Rogers TL, and Brown MV. 2013. The gut bacterial community of mammals from marine and terrestrial habitats. PLOS ONE 8:e83655.

Parks DH, Tyson GW, Hugenholtz P, and Beiko RG. 2014. STAMP: statistical analysis of taxonomic and functional profiles. Bioinformatics 30:3123-3124.

Parte A, Krieg NR, Ludwig W, Whitman WB, Hedlund BP, Paster BJ, Staley JT, Ward N, and Brown D. 2011. Bergey's Manual of Systematic Bacteriology: Volume 4: The Bacteroidetes, Spirochaetes, Tenericutes (Mollicutes), Acidobacteria, Fibrobacteres, Fusobacteria, Dictyoglomi, Gemmatimonadetes, Lentisphaerae, Verrucomicrobia, Chlamydiae, and Planctomycetes: Springer New York.

Phayungsak M, Phimsucha B, Wanilada R, Sopacha A, Sirawut K, Piamsak M, and Sage C. 2018. Bacterial community composition and distribution in different segments of the gastrointestinal tract of wild-caught adult Penaeus monodon. Aquaculture Research 49:378-392. doi:10.1111/are.13468

Pruesse E, Peplies J, and Glöckner FO. 2012. SINA: accurate high-throughput multiple sequence alignment of ribosomal RNA genes. Bioinformatics 28:1823-1829. 
571 Pruesse E, Quast C, Knittel K, Fuchs BM, Ludwig W, Peplies J, and Glöckner FO. 2007.

572

573

574

575

576

577

578

579

580

581

582

583

584

585

586

587

588

589

590

591

592

593

594

595

596

597

598

599

600

601

602

603

604

605

606

607

608

609

610

611

612

613

614

615

616
SILVA: a comprehensive online resource for quality checked and aligned ribosomal RNA sequence data compatible with ARB. Nucleic Acids Research 35:7188-7196.

Rungrassamee W, Klanchui A, Maibunkaew S, and Karoonuthaisiri N. 2016. Bacterial dynamics in intestines of the black tiger shrimp and the Pacific white shrimp during Vibrio harveyi exposure. Journal of invertebrate pathology 133:12-19.

Saffran K, Cash K, Hallard K, Neary B, and Wright R. 2001. Canadian water quality guidelines for the protection of aquatic life. CCME water quality Index 1:34-31.

Schock TB, Duke J, Goodson A, Weldon D, Brunson J, Leffler JW, and Bearden DW. 2013. Evaluation of Pacific White Shrimp (Litopenaeus vannamei) Health during a Superintensive Aquaculture Growout Using NMR-Based Metabolomics. PLOS ONE 8:e59521. 10.1371/journal.pone.0059521

Tall A, Hervio-Heath D, Teillon A, Boisset-Helbert C, Delesmont R, Bodilis J, and Touron-Bodilis A. 2013. Diversity of Vibrio spp. isolated at ambient environmental temperature in the Eastern English Channel as determined by pyrH sequencing. Journal of applied microbiology 114:1713-1724.

Tang Y, Tao P, Tan J, Mu H, Peng L, Yang D, Tong S, and Chen L. 2014. Identification of bacterial community composition in freshwater aquaculture system farming of Litopenaeus vannamei reveals distinct temperature-driven patterns. International journal of molecular sciences 15:13663-13680.

Thompson F, Gevers D, Thompson C, Dawyndt P, Naser S, Hoste B, Munn C, and Swings J. 2005. Phylogeny and molecular identification of vibrios on the basis of multilocus sequence analysis. Applied and Environmental Microbiology 71:5107-5115.

Tran L, Nunan L, Redman RM, Mohney LL, Pantoja CR, Fitzsimmons K, and Lightner DV. 2013. Determination of the infectious nature of the agent of acute hepatopancreatic necrosis syndrome affecting penaeid shrimp. Diseases of aquatic organisms 105:45-55.

Tremblay J, Singh K, Fern A, Kirton E, He S, Woyke T, Lee J, Chen F, Dangl J, and Tringe S. 2015. Primer and platform effects on 16S rRNA tag sequencing. Frontiers in Microbiology 6. 10.3389/fmicb.2015.00771

Utter DR, Mark Welch JL, and Borisy GG. 2016. Individuality, Stability, and Variability of the Plaque Microbiome. Frontiers in Microbiology 7. 10.3389/fmicb.2016.00564

Wang F-I, and Chen J-C. 2006. Effect of salinity on the immune response of tiger shrimp Penaeus monodon and its susceptibility to Photobacterium damselae subsp. damselae. Fish \& shellfish immunology 20:671-681.

Watts MP, Spurr LP, Gan HM, and Moreau JW. 2017. Characterization of an autotrophic bioreactor microbial consortium degrading thiocyanate. Applied microbiology and biotechnology 101:5889-5901. 10.1007/s00253-017-8313-6

Wickham H, and Wickham MH. 2007. The ggplot package.

Wright JJ, Konwar KM, and Hallam SJ. 2012. Microbial ecology of expanding oxygen minimum zones. Nature reviews Microbiology 10:381.

Wyban J, Walsh WA, and Godin DM. 1995. Temperature effects on growth, feeding rate and feed conversion of the Pacific white shrimp (Penaeus vannamei). Aquaculture 138:267279. https://doi.org/10.1016/0044-8486(95)00032-1

Xiong J, Wang K, Wu J, Qiuqian L, Yang K, Qian Y, and Zhang D. 2015. Changes in intestinal bacterial communities are closely associated with shrimp disease severity. Applied microbiology and biotechnology 99:6911-6919. 
617 Xiong J, Zhu J, Dai W, Dong C, Qiu Q, and Li C. 2017. Integrating gut microbiota immaturity 618 and disease-discriminatory taxa to diagnose the initiation and severity of shrimp disease. 619 Environmental Microbiology 19:1490-1501.

620 Yoon SH, Ha SM, Kwon S, Lim J, Kim Y, Seo H, and Chun J. 2017. Introducing EzBioCloud: a 621 taxonomically united database of 16S rRNA gene sequences and whole-genome $622 \quad$ assemblies. Int J Syst Evol Microbiol 67:1613-1617. intestinal microbiota in pacific white shrimp (Litopenaeus vannamei) at different culture stages. PeerJ 5:e3986.

Zhang M, Sun Y, Chen K, Yu N, Zhou Z, Chen L, Du Z, and Li E. 2014. Characterization of the intestinal microbiota in Pacific white shrimp, Litopenaeus vannamei, fed diets with different lipid sources. Aquaculture 434:449-455.

Zhu J, Dai W, Qiu Q, Dong C, Zhang J, and Xiong J. 2016. Contrasting ecological processes and functional compositions between intestinal bacterial community in healthy and diseased shrimp. Microbial ecology 72:975-985.

632

633 


\section{Table $\mathbf{1}$ (on next page)}

Summary of field collection and sampling design 
1 Table 1: Summary of field collection and sampling design

\begin{tabular}{|c|c|c|c|c|c|c|}
\hline Farm & $\begin{array}{l}\text { \# Pond } \\
\text { (Replicate } \\
\text { per pond) }\end{array}$ & $\begin{array}{l}\text { \# Shrimp } \\
\text { Sampled }\end{array}$ & Location & $\begin{array}{l}\text { Collection } \\
\text { Date }\end{array}$ & $\begin{array}{c}\text { Mean } \\
\text { Temperature } \\
\left({ }^{\circ} \mathrm{C}\right)\end{array}$ & $\begin{array}{c}\text { Reported } \\
\text { Age } \\
\text { (days post } \\
\text { hatching) }\end{array}$ \\
\hline $\begin{array}{l}\text { Marine Research } \\
\text { Station of } \\
\text { Fisheries and } \\
\text { Technical }\end{array}$ & $4(2-3)$ & 6 & $\begin{array}{l}\text { Quang Yen, Quang Nihn, } \\
\text { Vietnam }\end{array}$ & $\begin{array}{l}\text { December } \\
2015\end{array}$ & 20 & 97 \\
\hline Private & $1(4)$ & 4 & $\begin{array}{l}\text { Quang Yen, Quang Nihn, } \\
\text { Vietnam }\end{array}$ & $\begin{array}{l}\text { December } \\
2015\end{array}$ & 20 & 115 \\
\hline Private & $9(2-3)$ & 14 & Sitiawan, Perak, Malaysia & $\begin{array}{l}\text { March } \\
2016\end{array}$ & 30 & 65 \\
\hline
\end{tabular}

2 


\section{Figure 1}

Distribution of abundant phyla in all samples.

Significantly abundant phyla are annotated at the legends. Phyla abundance of pond water replicates were collapsed according to the location of sampling (see Supplementary Table 1). Sample type (MF, Malaysian rearing water; VF, Vietnamese rearing water; MS, Malaysian shrimp; VS, Vietnamese shrimp) with significantly higher phylum abundance than that of another group (Superscript) were shown in bracket next to their associated phylum legend. For example, Actinobacteria (MF*; $\mathrm{VF}^{\mathrm{vs}, \mathrm{Ms}}$ ) indicates that this phylum is significantly more abundant in Malaysian rearing water samples compared to all three other groups and that it is more abundant in Vietnamese rearing water samples compared to shrimp samples from both countries. Hash and asterisk signs next to $y$-axis labels indicate mussel-infested and suspected diseased samples, respectively. 


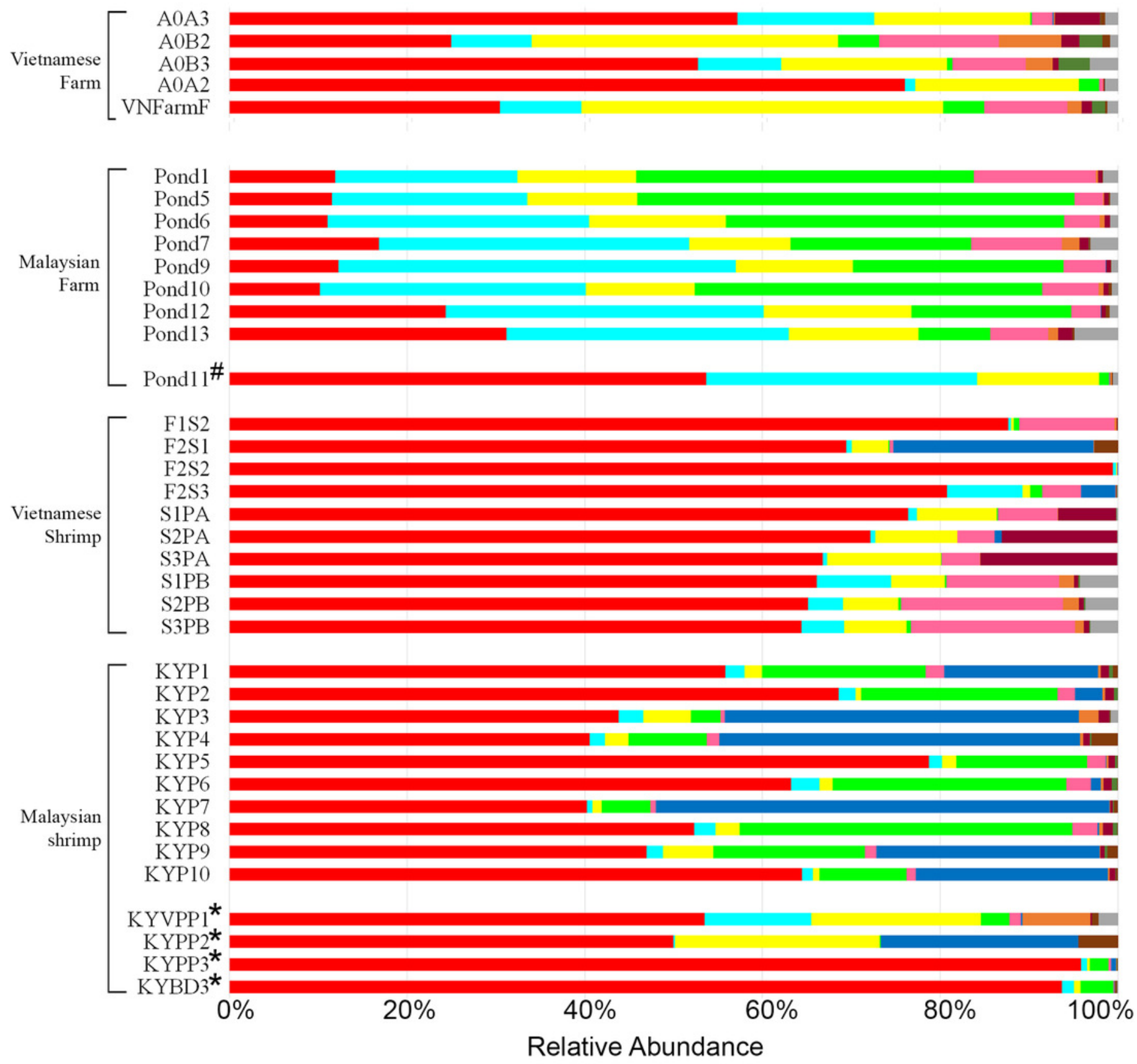

\begin{tabular}{|c|c|c|}
\hline - Proteobacteria $\left(\mathrm{VS}^{\mathrm{MF}, \mathrm{VF}} ; \mathrm{MS}^{\mathrm{MF}, \mathrm{VF}} ; \mathrm{VF}^{\mathrm{MF}}\right.$ ) & Actinobacteria $\left(\mathrm{MF}^{*} ; \mathrm{VF}^{\mathrm{Ms}} \mathrm{vs}\right)$ & Bacteroidetes $\left(\mathrm{VF}^{\circ} ; \mathrm{MF}^{\mathrm{Ms}, \mathrm{vs}}\right)$ \\
\hline Cyanobacteria $\left(\mathrm{MF}^{*} ; \mathrm{MS}^{\mathrm{vs}}\right)$ & Planctomycetes & - Fusobacteria $\left(\mathrm{MS}^{*}\right)$ \\
\hline Patescibacteria & - Verrucomicrobia & Chloroflexi \\
\hline Firmicutes & others & \\
\hline
\end{tabular}


Figure 2

Rarefaction curves and alpha diversity plots of each sample group.

(A) Rarefaction curve constructed based on observed ASVs (B) Rarefaction curve constructed based on phylogenetic distance (PD_whole_tree) (C) Shannon's evenness index box plot (D) Shannon index box plot.

A

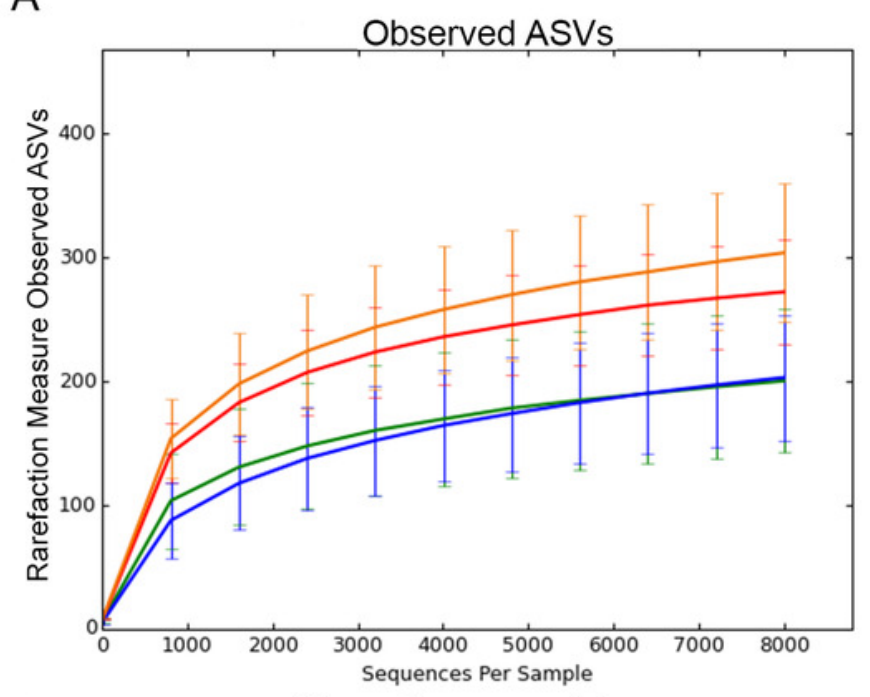

C

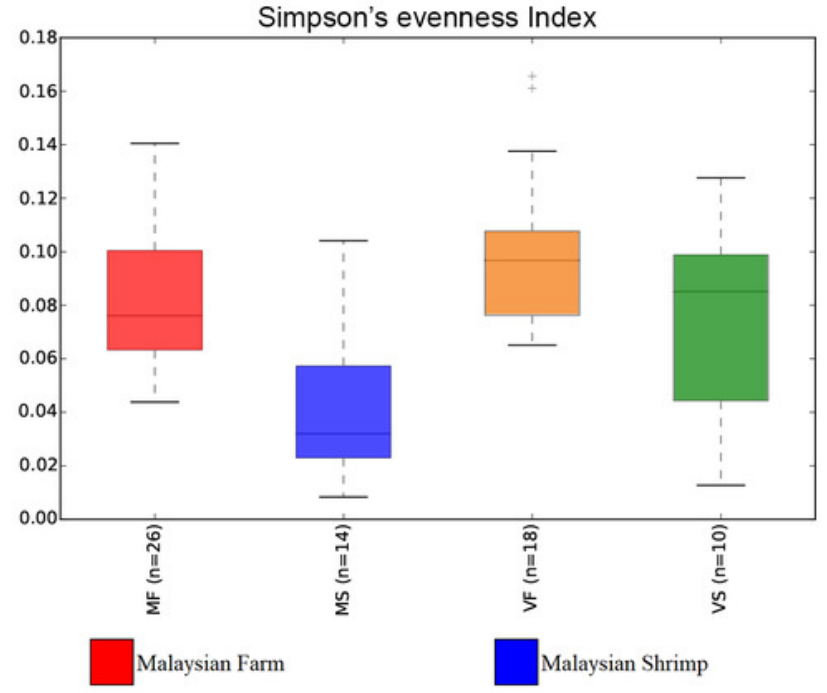

B

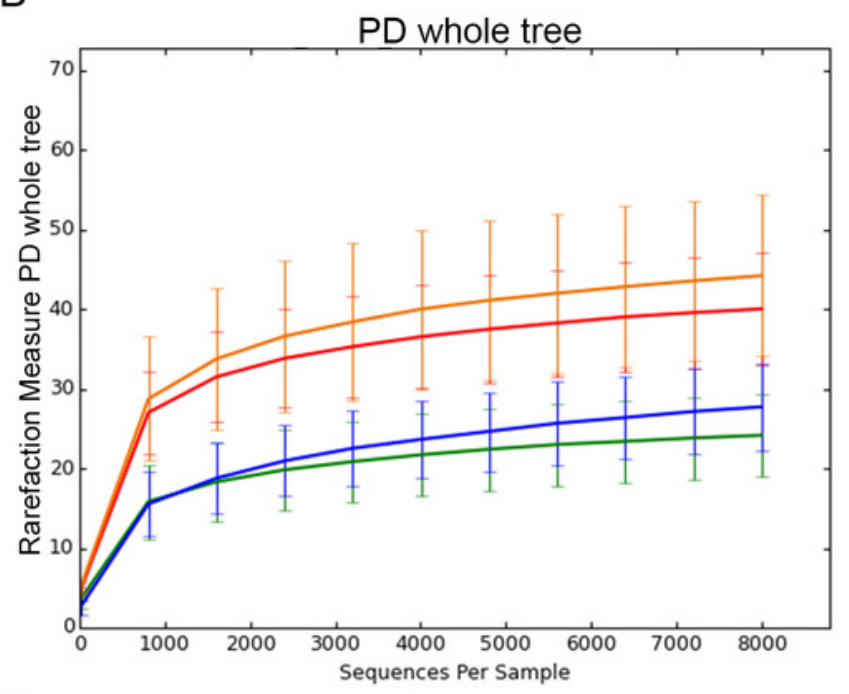

D

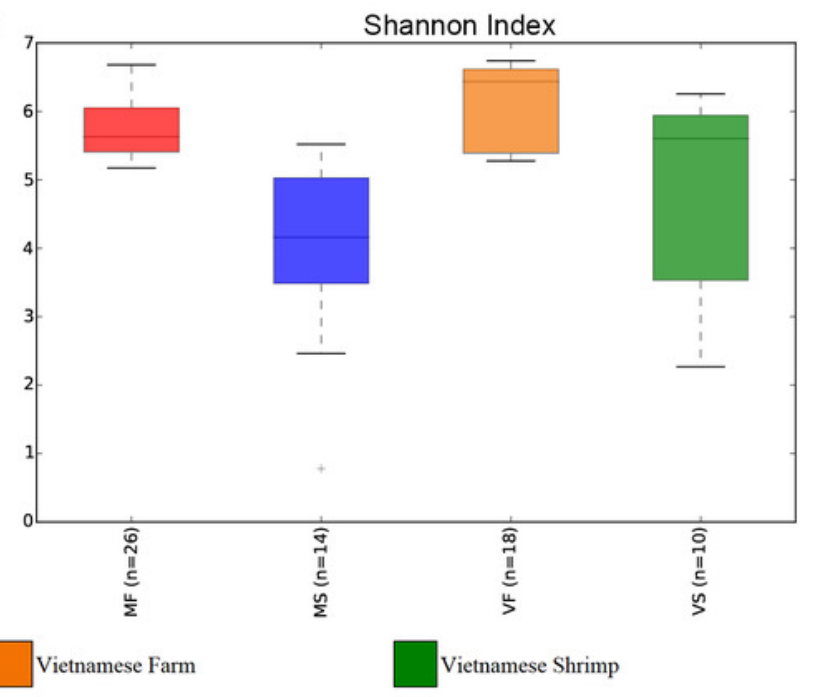




\section{Figure 3}

Principal component analysis of (A) weighted Unifrac and (B) unweighted Unifrac distances.

Red circular outline shows 'Malaysian pond' cluster, yellow circular outline shows 'Vietnamese pond' cluster, blue circular outline marks 'Malaysian shrimp' cluster, and green circular outline represents 'Vietnamese shrimp sample. The sample points were coloured according to "Country_Source_Location" information (Supplementary Table 1). Country and source information of each points were abbreviated; for example, Malaysian farm sample from Pond 1 was labelled as MF_Pond1.

A
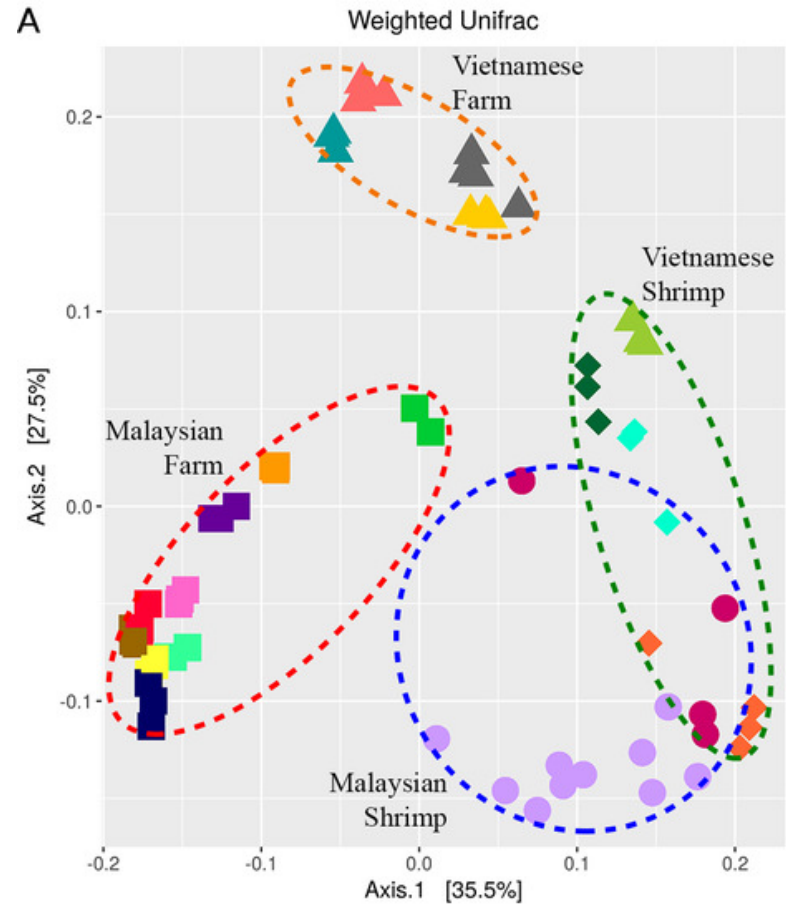

B
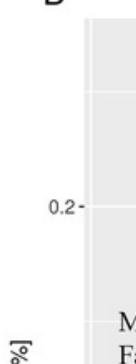

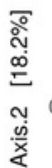
$0.0 \cdot$

Malaysian $0.0-i$

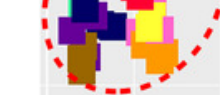

$-0.2-$

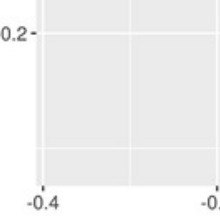

$\begin{array}{ll} & 0.0 \\ \text { Axis. } 1 & {[33.5 \%]}\end{array}$

Unweighted Unifrac

Malaysian

Shrimp
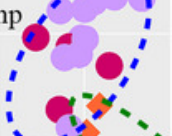

- - ?
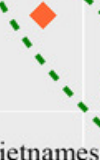

Shrimp
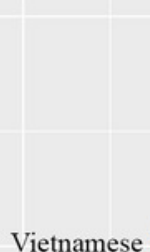

ietnamese

Farm

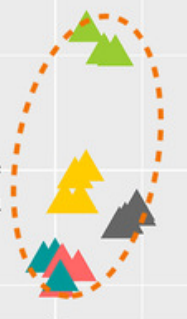

Country_Source_Location

MF_Pond1

MF_Pond10

MF_Pond1

MF_Pond12

MF_Pond13

MF Pond5

MF_Pond6

MF_Pond7

MF_Pond9

MS

MS_DiseasedShrimp

$\triangle V F$ A0A2

VF_A0A3

$\triangle V F+{ }_{\text {A0B2 }}$

$\triangle \mathrm{VF}_{-} \mathrm{AOB3}$

$\triangle$ VF_VNFarmF

vS_AOA3

VS_AOB3

vs_vNFarms 


\section{Figure 4}

Microbial community dynamics at the family level

(A) Venn diagram illustrating the number of unique and overlapping microbial families among shrimp guts and rearing water. To be considered as present, a microbial family must be detected in at least $90 \%$ of the samples from the same group. (B) Tukey post-hoc pairwise comparison in conjunction with analysis of variance (ANOVA) between "Country_Source" groups of nine significant microbial families. Mean proportion values of families that are significantly different are shown in the bar plots on the left section, while the differences in the pairwise comparison mean proportion with $95 \%$ confidence interval are shown on the right section. 


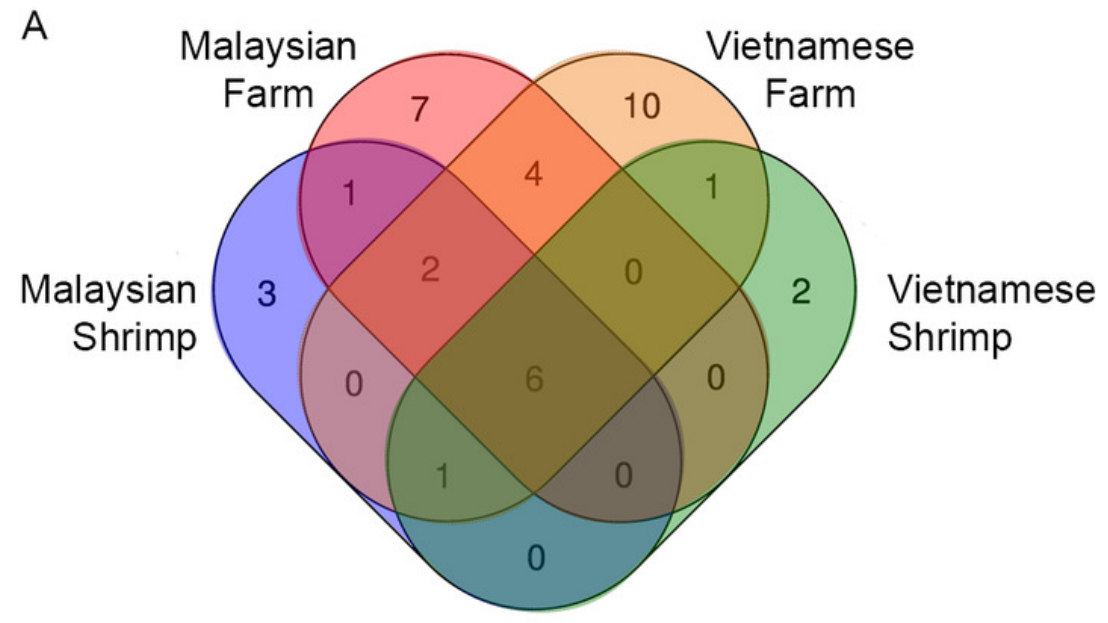

B

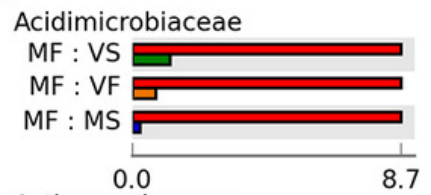

Actinomarinaceae

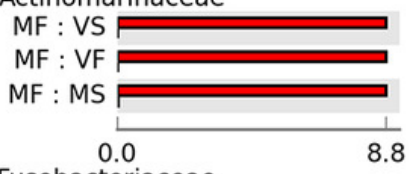

Fusobacteriaceae

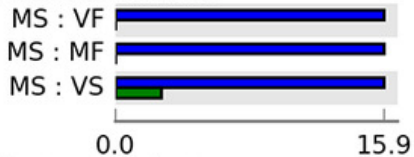

Comamonadaceae

VF : VS

VF : MS

VF : MF

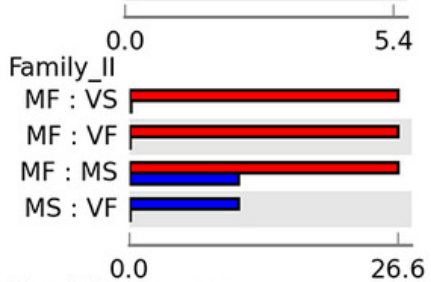

Rhodobacteraceae

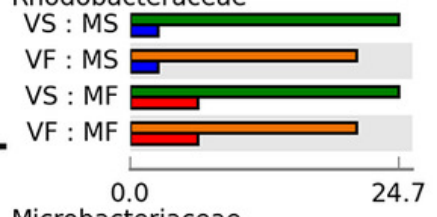

Microbacteriaceae

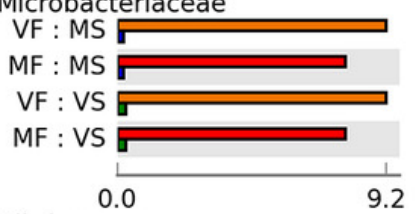

Vibrionaceae
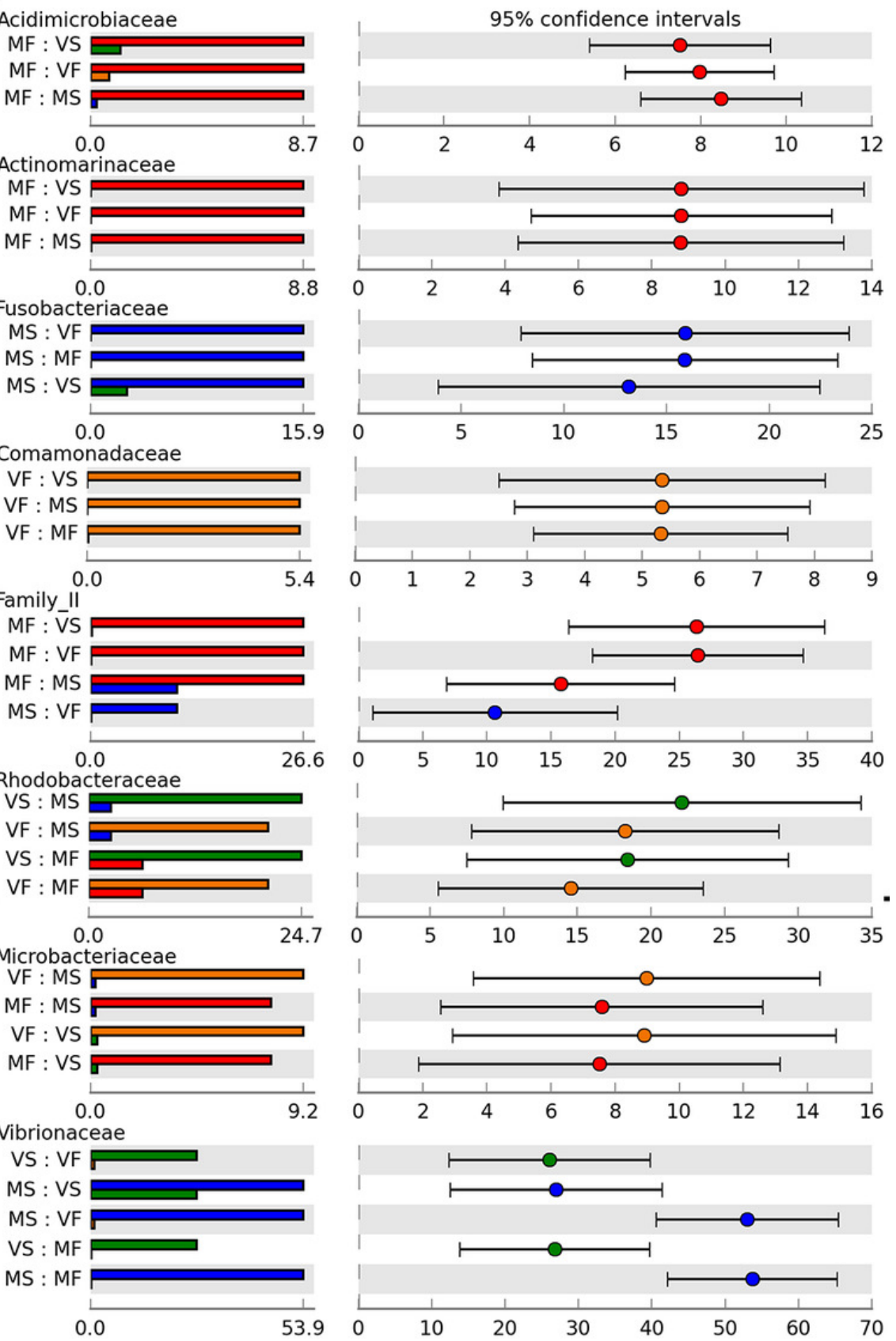

Mean proportion $(\%) \quad$ Difference in mean proportions (\%)
Peerj reviewing PDF |(2018:06:28940:2:0:REVIEW 27 Aug 2018)

Malaysian Farm 


\section{Figure 5}

Abundance and prevalence of core genera in shrimp guts.

(A) Normalized read counts of the selected genera among Malaysian and Vietnamese shrimp gut samples (B) Percentage of shrimp gut samples harbouring the core genera

A

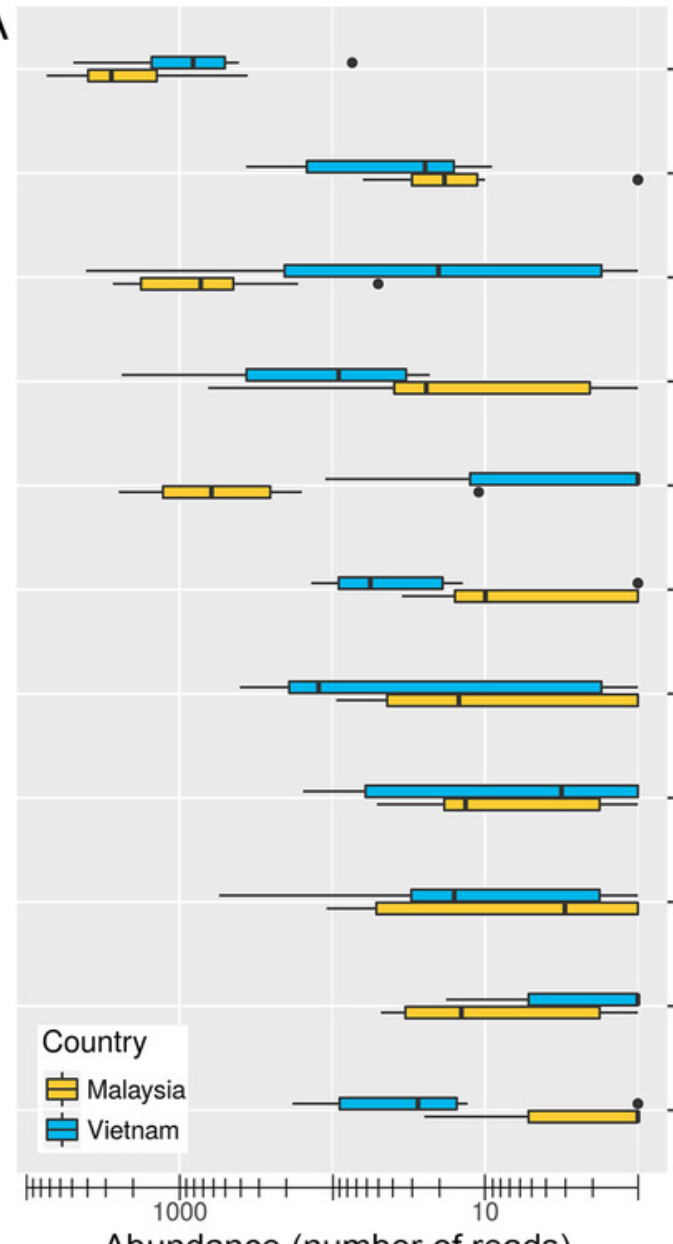

Abundance (number of reads)

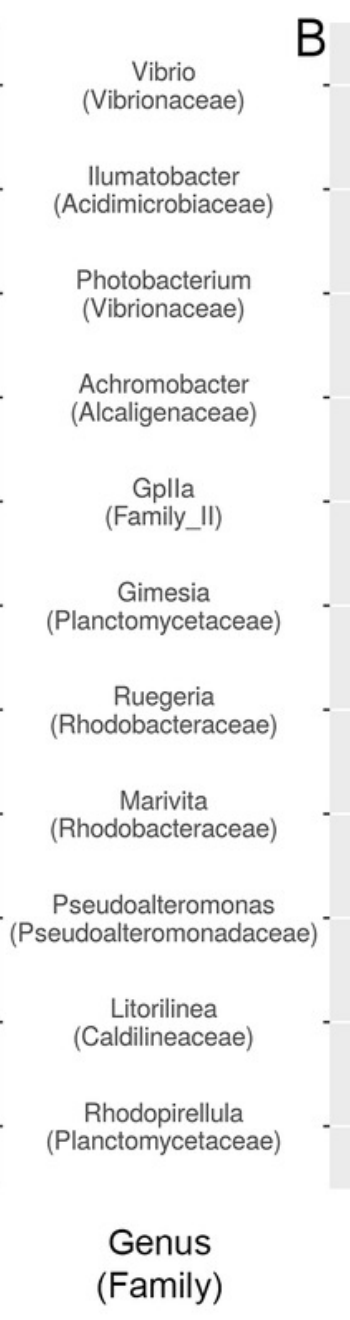

B
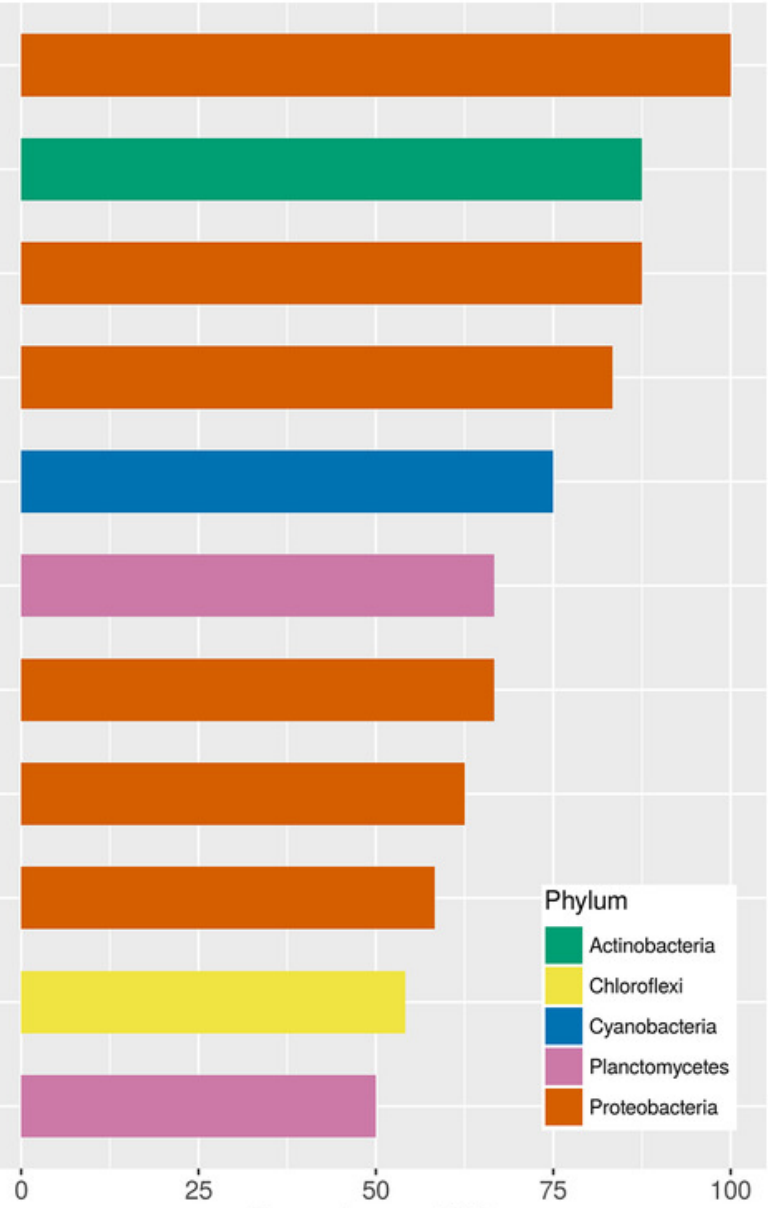

Prevalence (\%) 
Figure 6

Cumulative normalized read counts of OTUs/ASVs classified as Vibrio and similarity table against selected type-strain Vibrio sequences

Empty and filled bars indicate shrimp gut and rearing water samples, respectively. Different

Vibrio groups consisting of one OTU and its associated ASVs were separated by grey

horizontal lines.

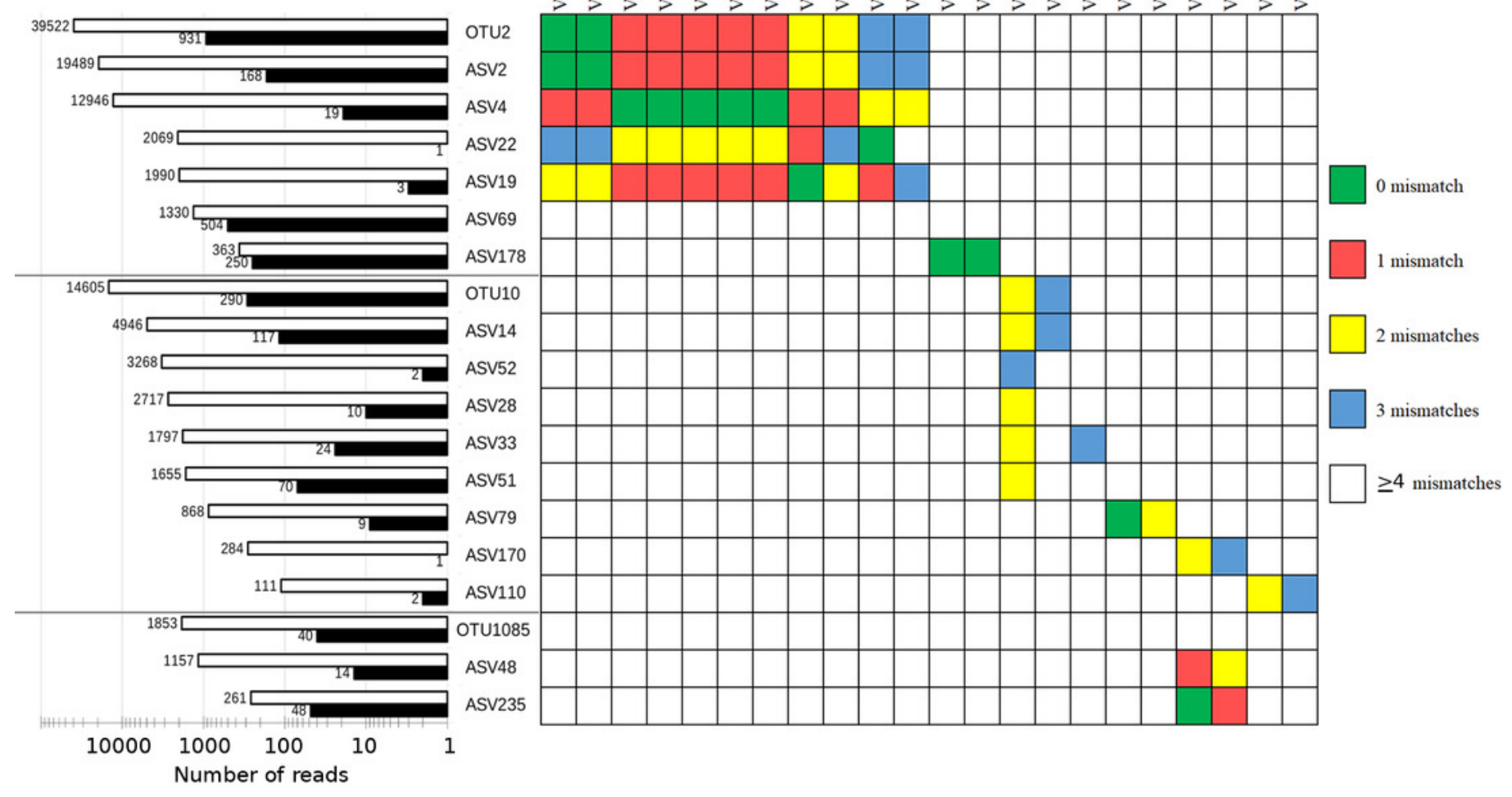

